\section{Aronsky, C.F. Aliferis, K.B. Johnson, N. Lorenzi, R.A. Miller}

Dept. of Biomedical Informatics

Eskind Biomedical Library

Nashville, Tennessee, USA

\title{
Research and Education
}

\section{Biomedical Informatics Training Program at Vanderbilt University}

In addition, there is a strong component comprised of organizational theory and applications, tailored to health-care systems and institutions. Biomedical informatics extends beyond the narrow focus of biomedical computer systems design, application, and evaluation, by providing theory and tools for approaching both health-related processes and research from an analytical and rational perspective. This is exemplified by its foundations in studies involving clinical problem-solving, research on improving diagnosis and therapy, the analysis of clinicians' information needs, and its emphasis on solutions that embody the evidencebased practice framework.

The environment for training in biomedical informatics at Vanderbilt University has evolved substantially over the past decade. Now supported by a training grant from the National Library of Medicine, opportunities involve Vanderbilt University Medical Center, the Middle Tennessee Veterans Administration facilities, and Meharry Medical College as potential training sites. Training occurs at multiple levels: informal and formal undergraduate/medical school elective rotations in biomedical informatics (including summerinternships), formal graduate study leading to a masters or doctoral degree, and post-doctoral nondegree fellowship training. Trainees may focus on healthcare informatics, bioinformatics, or a combination of the two. This article describes the evolution, infrastructure, and activities related to the current training program in Biomedical Informatics at Vanderbilt.

\section{The VUMC Informatics Center}

In 1991, William W. Stead, MD, was recruited to Vanderbilt University Medical Center (VUMC) as Associate Vice Chancellor for Health Affairs, Director of the Informatics Center, and Professor of Medicine. The Informatics Center was formed as a consequence of Dr. Stead's recruitment. By 1993, the Informatics Center initially consisted of the Division of Biomedical Informatics (DBMI), the soon-to-be constructed Irwin and Annette Eskind Biomedical Library, and the hospital-and-clinic-based applied information technology group. As an interdisciplinary center within the University (as opposed to an academic unit within the School of Medicine), the Informatics Center was designed to provide space for visiting researchers from other Vanderbilt departments, and for visiting professors from other institutions, and has done so since its inception.

Unique among academic health centers at the time (during the 1990s), VUMC entrusted the Informatics 
Center with the responsibility for: (a) providing the essential information infrastructure for patient care, management, research and education, and including the support for informaticsrelated research and education in clinical informatics and the emerging field of bioinformatics (including new initiatives in genomics and proteomics); and, (b) fusing scholarly research in biomedical informatics with the dissemination of the resultant knowledge to individuals through its education programs and into operation through the infrastructure.

Under Dr. Stead's leadership, members of all of the units of the Informatics Center have been linked into a cohesive team with diverse talents. The Informatics Center has collectively had responsibility for software acquisition, development and maintenance within VUMC clinical facilities; corresponding hardware acquisition and maintenance tasks; network hardware, software, and maintenance; and, project management. All the Informatics Center units have collaborated closely, so that academic software projects, once mature, can be delivered directly to hospital wards or clinics in conjunction with programmers and managers from the hospital and clinics - the individuals who are responsible for the longterm maintenance of such projects. Conversely, the applied hospital-andclinic-based IT group has involved members of the academic unit (DBMI) directly in its leadership infrastructure, planning, development, and troubleshooting activities. This synergy within the Informatics Centers has created an unparalleled laboratory for informatics research, fostering multidisciplinary rapid prototyping and deployment of applied systems, and the evaluation of their impact. Researchers have had access to real-world problems and operational monies (the Informatics Center budget averaged more than twenty-five million dollars per annum over its first decade). Academic faculty members have been encouraged to meld informatics service responsibilities with informatics research activities in a manner that leverages the clinical and research facilities of the university as an "applied informatics" laboratory.

\section{Vanderbilt University Medical Center's role as a DBMI "Informatics Laboratory"}

The Informatics Center's integration of service responsibilities with research opportunities has served as a mechanism to align faculty research interests with the strategic initiatives of the medical center. Academic trainees learn how to exploit knowledge from a rigorous academic program in order to design, implement, and evaluate real-world informatics applications. The medical center continues to benefit from a technology infrastructure and architecture that takes full advantage of the new science of biomedical informatics, in order to achieve a scale of productivity not possible with traditional information systems. The VUMC's daily functions depend on systems built and maintained by DBMI faculty and trainees. The following series of system descriptions give a sample of the breadth of opportunities for research and evaluation available to trainees.

The Vanderbilt Care Provider Order Entry (CPOE) system integrates clinical decision support with the process of order generation and communication for VanderbiltUniversity Hospital orders on all inpatients, including the emergency department. The Vanderbilt CPOE system has been continuously developed by DBMI faculty working with Informatics Center staff and trainees at Vanderbilt since 1994. Its major developer, Dr. Antoine Geissbühler, initially created the system as an applied postdoctoral fellowship project in 1994-96, working with Dr. Miller and Dr. Stead. The CPOE system is used "at the point of care" to support clinicians as they make important decisions regarding a patient's clinical course - it is not the more typical sort of clerical order entry system. The CPOE system helps to ensure the highest quality of patient care, while reducing medical errors. Over 12,000 orders are entered into the CPOE system daily. The CPOE system components include an "intelligent, heads-up display" approach to patient care that provides clinicians with information they need to know when they need to know it. The CPOE system has an interface to the clinical data repository so that both order-related and lab-related alerts can be generated. The CPOE system is sensitive to patients' specific information and provides: medication prescription with safeguards including allergy alerts, dose checking for selected medications, drug interaction alerts, and FDA alerts (or withdrawal notices) for drugs; flexible tools to present and activate guidelines through a scripting language developed for use by non-programmers; implementation of "Best of Care" clinical pathways through over 900 individual "order sets" that are maintained by clinical experts in relevant specialty fields; representation of individual physicians' preferences; hooks to web-based "justin-time" educational resources, such as drug monographs and contextspecific educational Web pages; and, linkage of patient cases to literaturebased evidence through use of the NLM UMLS Knowledge Sources and a link to NLM's PubMed bibliographic search engine. Clinical alerts lead to appropriate warnings for allergies, drug-drug interactions, and for substitution of one drug for another drug, as recommended by the institutional 
Pharmacy and Therapeutics Committee. Currently, DBMI faculty members Russ Waitman, PhD, and Randolph A. Miller, MD, are responsible for the CPOE system's development and maintenance. They work regularly with a team of programmers, clinical data/information/ knowledge managers (including informatics trainees), and hospitaland-clinic based health care providers. Vanderbilt entered into a relationship with McKesson to commercialize the CPOE system.

StarChart is an integrated, longitudinal electronic patient record developed by Dario Giuse, DrIng, working with Informatics Center staff since 1998. The system receives data from sources as diverse as the VUMC interface engine (real-time lab results, radiology reports, etc.) and outside transcription companies. StarChart stores patient information permanently in a highly replicated distributed database system. Documents from the disparate data sources are parsed, converted into an internal XML-like format and immediately replicated to three sets of database servers located in different geographical locations (including the non-medical Vanderbilt University data center). The StarChart database is optimized to support the most common forms of clinical queries. Efficient, word-level indexing of each record also supports extensive crosspatient search capabilities. All clinical, textual, and numerical patient data generated at VUMC over the past decade have been stored in StarChart, with the exception of inpatient and outpatient progress notes, most of which are handwritten. In recent years, StarChart has been expanded to create customized groups or "panels" of patients (StarPanel), that are of particular interest to a clinician, such as patients that the clinician has seen in primary care outpatient clinic, or patients currently admitted to the hospital ward on which the doctor is the "teaching" attending physician. StarPanel alerts clinicians to new information garnered anywhere in the health center regarding patients in their personal "panels," and especially facilitates care of outpatients.

Vanderbilt has multiple clinical environments in which informatics researchers and trainees can develop and deploy useful tools. One such effort is the E3 project ("electronic outpatient processes by 2003"). This project, spearheaded by Nancy Lorenzi, $\mathrm{PhD}$, started in early 2000 when VUMC decided to implement StarChart and StarPanel as the electronic health record for all outpatient visits (approximately 700,000 peryear). The goal of E3 was to remove paperbased processes from more than 90 Vanderbilt Medical Group outpatient sites. The success of the E3 project provided many opportunities for student involvement; e.g., writing programs to enhance current electronic health record functions to create new functions; evaluating the impact of the electronic health record on patient care, safety, quality, or the organization; or, studying any number of clinical topics, for which a vast volume of information exists. In light of the success of the E3 project, the E5 project was born. The E5 project focuses on reducing paperbased processes in the inpatient setting based on extensions to the informatics tools that were developed for the outpatient area.

Judy Ozbolt, PhD, leads the EMILIE (Electronically Mediated Interdisciplinary Learning in an IntegratedEnvironment) project, which supports continual quality improvement in patient care and is funded in part by a grant from the National Library of Medicine. The EMILIE software developed by DBMI faculty and trainees (the CPOE Admission Wizard) assists physicians in using the CPOE system to select an appropriate evidence-based order set as the starting point for developing customized orders for each patient. These order sets are coordinated with interdisciplinary collaborative care pathways contained in the PathworX care management system that nurses use to plan and organize patient care, and to track achievement of therapeutic goals. A group of faculty and trainees are evaluating the effects of the CPOE Admission Wizard on selection of pathway-related order sets, compliance with order sets, achievement of therapeutic goals, patient outcomes, and costs.

The Emergency Department represents an especially informationintensive environment. In an effort to facilitate more efficient communication and information management within the emergency department at Vanderbilt, Dominik Aronsky, MD, PhD, working with clinicians, Informatics Center developers, and DBMI trainees, developed an Emergency Department information system that was implemented in 2002. A computerized whiteboard, including a large touch-sensitive plasma monitor, is the core of the information system and provides an overview of the patient's location, patient care related information and the current emergency department activity. The whiteboard has become an indispensable tool for managing patients and the overall emergency department operation. The whiteboard represents the emergency department's primary portal for obtaining additional operational and patient care information. As the whiteboard is embedded into StarChart, the institution's computerized patient record, emergency department staff has fast access to the information of individual patients. The generation of regular and ad-hoc reports allows the emergency department to examine operational activities, 
monitor educational goals in residency training, participate in biosurveillance programs by state and national agencies, and provide data for research activities.

The Discovery Systems Laboratory (DSL), led by Constantin Aliferis, $\mathrm{MD}, \mathrm{PhD}$, is a component of DBMI that reaches beyond the School of Medicine. It has been funded in part since 2001 through support from the Academic Venture Fund of Vanderbilt University. The broad mission of the DSL is to develop, evaluate and deploy innovative algorithms and systems for biomedical discovery and decision making. The DSL currently has 3 dedicated faculty, 8 collaborating faculty, 7 graduate students, and 4 staff members. Since its inception, the DSL faculty and staff have developed innovative computational methods in the following areas: (1) Algorithms for variable (feature) selection for classification: DSL members have developed a family of Markov Blanketbased discovery algorithms that are computationally tractable, sound, and sample efficient at the same time. These algorithms can be used for optimal variable selection for classification in several domains, such as gene expression diagnosis, mass spectrometry diagnosis, clinical diagnosis, or text categorization. (2) Algorithms for induction of causal theories from data: DSL researchers have developed highly-scalable and sound algorithms for causal discovery that are currently being investigated (with funding from the NIH) for discovery of genes that cause lung cancer and epidemiological factors responsible for breast cancer. (3) Models and systems to support Evidence-Based Practice. DSL researchers have developed text categorization models that classify medical articles according to the methodological criteria of the ACP Journal Club. (4)Computationalmodels of lung cancer. In addition to discovery of genes affecting tumorigenesis in the lung, DSL research also deals with constructing: diagnostic models for distinguishing among different types of cancer, between normal controls and patients, between metastatic and nonmetastatic tumors; prediction of response to treatment; construction of new molecular types of lung cancer; and combined gene-expression/ spectrometry/clinical datasets for diagnosis and prognosis. Other areas of DSL research include theoretical and empirical properties of learning algorithms, basic research in planning, and the development of tools to support gene expression-based diagnosis. Additional information about the laboratory's activities can be found at: http://discover1.mc.vanderbilt.edu/ discover/public/

Microarray technology, which facilitates the measurement of the relative levels of gene expression through a high-density parallel approach, has had a tremendous impact on biomedical research by providing an unprecedented view of the transcriptional state of a cell or tissue on a genome scale. The Vanderbilt Microarray Shared Resource (VMSR), directed by Shawn Levy, $\mathrm{PhD}$, was founded in 2000 to advance gene expression profiling and related informatics technologies while providing the highest quality technical service, comprehensive analysis, and statistical support. The VMSR, centrally located on the VUMC campus, offers full technical services ranging from RNA quality control, RNA amplification, RNA labeling and hybridization to a variety of microarray platforms. The VMSR facility is equipped to perform expression analysis using custom cDNA and oligonucleotide microarrays as well as the Affymetrix GeneChip and Amersham CodeLink platforms. The computing resources are networked to four central Unix servers providing both database storage as well as high-level statistical and analysis tools. Using high-throughputPCR and robotic liquid handling equipment the VMSR offers mouse microarrays generated from over 55,000 mouse cDNA clones from a number of libraries including the National Institute on Aging(NIA) 22.4k mouse cDNA set and the RIKEN FANTOM1 cDNA collection. The VMSR provides cDNA libraries to the academic community and is the largest distributor of the NIA clone sets and one of very few academic institutions to offer the RIKEN collection. Human microarrays are produced from a collection of over 11,000 cDNA clones and 31,000 oligonucleotides representing 28,000 human transcripts. Two full-time bioinformaticists provide training and support in all aspects of data analysis from image analysis through the statistical evaluation of the gene expression profiles. All data sets are available for analysis using web-based tools with other desktop tools including Affymetrix's Data Mining Tool, GeneSpring multi-array analysis software (Silicon Genetics), Insightful's S+ statistical package and ArrayAnalyzer module. Other academic tools such as SAM, PAM, Cluster and TreeView microarray data visualization programs (Stanford University), as well as custom developed statistical and data mining tools are also supported. Overall the VMSR operates under a comprehensive service model that begins with experimental design consultation and continues through data analysis and statistical support, providing investigators with efficient and fully supported access to these technologies.

Under the leadership of Nunzia Giuse, MD, MLS, a physician-librarianinformatician, the Eskind Biomedical Library prepares librarians to meet the challenges of current and future health care environments in innovative 
ways. The Clinical Informatics Consult Service (CICS) provides a direct link between information and clinical practice by promoting integration of librarians and interns with clinical teams, and was the basis for the current national focus on developing "informationists" as a profession. Each CICS librarian participates weekly in the morning rounds of a specific clinical unit. EBL librarians are a true part of the clinical team, participating much as clinical pharmacists do, as expert consultants and as information resources. This creates an environment where evidence-based medicine forms a part of daily life, and information is viewed in the vivid context of actual patient cases. Participants undergo continuous in-depth training in medical terminology, practice, and treatment, as well as tenets of evidence-based medicine. Strong emphasis is placed on the acquisition of medical knowledge, both in a broad spectrum and in a specific area (such as hematology/ oncology or trauma), which provides a basis for understanding clinical discussions and responding to clinical queries. Where the word "filtering" has traditionally been applied only to the literature search, CICS librarians go beyond this step to read and filter the full text. They synthesize, summarize, and present information relevant to the particular case at their unit's rounds, much as residents present their clinical cases to the team. Similarly, the Research Informatics Consult Service (RICS), begun in February of 1998 as an outgrowth of the CICS, focuses on supporting the needs of health sciences researchers. In addition to providing customized information, the RICS also analyzes researcher interests to develop proactive information alerts and to guide collection development. Services fall into the broad categories of Current Awareness, Training, Information Delivery, Grant Writing Assistance, Database Resources, Information Filtering, and
Comprehensive Database Searching. The RICS program has become a forum for researchers to request new library services or resources and offer feedback on the library's performance. The Patient Informatics Consult Service (PICS) aligns the library with the VUMC's goal of preeminent patient care. The PICS initiative extends the concept of librarians as information consultants to provide appropriate information to health care consumers, such as patients and their families. For this purpose, physicians are provided with "information prescription" pads, similar to the ones that are used to write prescriptions for medications. Using the information prescription pads physicians can communicate clearly the patient's information needs to the librarian. Librarians guide patients and family members in their search for information, considerably decreasing the risk that a patient or family member will find information irrelevant to their question. A copy of the information packet is also sent to the patient's clinician to improve the communication between clinicians and librarians as well as clinicians and patients.

\section{The Department of Biomedical Informatics}

In 1993, Dr. William Stead created, with the approval of the School of Medicine, the Division of Biomedical Informatics as a free-standing academic component of the School of Medicine. Dr. Randolph A. Miller was recruited as Chair of the Division of Biomedical Informatics, effective June 1, 1994. Dr. Miller previously was Professor of Medicine and Chief of the Section of Medical Informatics within the Division of General Medicine, in the Department of Medicine at the University of Pittsburgh School of Medicine (and founded the NIHfunded Training Program in Medical Informatics there). Dr. Miller recruited several colleagues from Pittsburgh to join him as Biomedical Informatics faculty at Vanderbilt: Dario Giuse, DrIng, who was on the faculty in the Robotics Institute at Carnegie Mellon University; Nunzia B. Giuse, MD, MLS, who was on the faculty in the Section of Medical Informatics at Pittsburgh; and, Antoine Geissbühler, MD, and Steven H. Brown, MD, who were post-doctoral fellows in the Section of Medical Informatics at Pittsburgh.

In 1999, Drs. Stead and Miller determined to add a "core" bioinformatics group to the growing DBMI faculty. As a result, Mary Edgerton, MD, PhD, Constantin Aliferis, MD, PhD, Ioannis Tsamardinos, $\mathrm{PhD}$, and Erik Boczko, $\mathrm{PhD}, \mathrm{PhD}$, joined the DBMI faculty during 2000-2002; the latter three are members of the new Discovery Systems Laboratory. In 2001, after substantial growth (13 faculty members) and considerable accomplishments, the Division was formally recognized as a Department within the School of Medicine.

The accomplishments of the DBMI faculty are substantial. Among the current faculty, there is one member of the Institute of Medicine, National Academy of Sciences (Stead); the founding and current editor (Stead, Miller) of the Journal of the American Medical Informatics Association, the major journal in Biomedical Informatics in the USA; five past or current presidents of major USA medical informatics-related organizations: the American Association for Medical Systems and Informatics (Stead), the American Medical Informatics Association (Miller); the American College of Medical Informatics (Stead, Miller); and the Medical Library Association (Lorenzi); and one president-elect of the International Medical Informatics Association (Lorenzi). 
Among the faculty are four past scientific program chairs of the AMIA Fall Symposium (formerly known as SCAMC), the major national meeting in Biomedical Informatics (Stead, Miller, Ozbolt, Lorenzi); and, two past chairs of the National Library of Medicine Study Section, which reviews federal grant proposals in Biomedical Informatics (Stead, Miller). The faculty includes past or present members of Editorial Boards of major biomedical informatics journals (Stead, Miller, Lorenzi, Ozbolt, N. Giuse, D. Giuse, Shultz, Johnson, Aronsky). Over the past nine years, the DBMI faculty has collectively received more than $\$ 15$ million in NIH grant support. Eight faculty members are elected Fellows in the 120-member honorary American College of Medical Informatics. A current listing of the DBMI faculty is listed in Appendix 1.

\section{Department of Biomedical Informatics Mission Statement}

The mission of the department consists of its:

Educational Mission: To educate undergraduate, graduate, and postgraduate trainees in the theory and practice of biomedical informatics;

Research and Service Missions: To develop and evaluate innovative techniques and technologies for the storage, retrieval, dissemination, and application of biomedical knowledge, in order to support clinical practice, research, life-long learning, and administration, and as a result, to contribute to the professional body of knowledge regarding biomedical informatics;

Professional and Ethical Missions: To maintain cooperation and collegiality with all those who learn and work with us locally, nationally, and internationally; and, to develop and disseminate ethical and professional standards for the conduct of biomedical informatics research and for the utilization and evaluation of health care informatics applications.

\section{The Biomedical Informatics Training Program}

With the arrival of Dr. Miller and several new faculty members in 1994, DBMI began to educate post-doctoral fellows interested in Biomedical Informatics, as well as graduate students interested in biomedical informatics who were seeking degrees in other Vanderbilt departments. In 2001, the university approved the department's proposal for a formal MS and $\mathrm{PhD}$ degree training program in Biomedical Informatics, which was prepared in large part by Dr. Aliferis (working in conjunction with Drs. Miller and Stead), who was also the program's founding director. In July 2002, the department began to receive substantial support for the education of trainees through a Training Grant from the National Library of Medicine.

The DBMI provides high-quality training for four groups of students:

(1) graduate degree candidates,

(2) post-doctoral fellows,

(3) students preparing for careers in information systems management (such as chief information officers), and

(4) students in training to be health care professionals (i.e., students enrolled in medical, nursing, dental or other similar programs.)

\section{Graduate Degree Program in Biomedical Informatics}

The DBMI degree program offers students an opportunity to pursue both $\mathrm{MS}$ and $\mathrm{PhD}$ degrees. As anticipated, candidates for these programs come from a wide variety of backgrounds, with generally superb skills, but with insufficiently deep skills in selected areas of the broad discipline of Biomedical Informatics. As a general guideline, DBMI students take at least two years to complete the MS degree and at least five (cumulative) years to complete the $\mathrm{PhD}$ degree; however, the program is constructed to accommodate students who require additional skills, or facilitate those with previous experience allowing them to advance through the program more quickly.

During the first two years of training, students focus on the foundational aspects of their training by taking didactic courses from three categories: 1) core biomedical informatics courses; 2) courses in three core areas of importance tobiomedicalinformatics training; and 3) electives. As a major emphasis of the training program and animportant supplement to the didactic course work, students are involved from the very beginning in ongoing clinical informatics and bioinformatics research projects.

Because the DBMI educational program must support the needs of trainees from a broad variety of backgrounds, DBMI supports trainees' participation in trans-institutional educational opportunities. As an example DBMI has partnered with an existing program at Vanderbilt, called the "Interdisciplinary Graduate Program." This program is a combined recruiting strategy for eight biomedical science departments. Students spend the first year in a shared introductory curriculum that provides an overview and the basic aspects of the participating departments. Three rotations of varying length allow students to choose three departments of closer interest to obtain specific research experience within a particular field. Students in the Interdisciplinary Graduate Program can select biomedical informatics for such a rotation and subsequently pursue the $\mathrm{PhD}$ in 
the department. In addition, DBMI students who have a particular interest in bioinformatics (as opposed to clinical informatics) can also participate in the Interdisciplinary Graduate Program for one or two semesters, providing indepth knowledge of basic biomedical science to supplement the bioinformatics related coursework.

\section{Core Biomedical Informatics Knowledge}

Five core biomedical informatics courses provide the basic knowledge in biomedical informatics and are listed in Table 1.

All courses are associated with a laboratory experience to help teach additional in-depth concepts in an interactive setting.

Table 1. Core Biomedical Informatics Courses

\begin{tabular}{|c|c|}
\hline Core Course & Course Summary \\
\hline $\begin{array}{l}\text { Foundations of Biomedical } \\
\text { Informatics }\end{array}$ & $\begin{array}{l}\text { An introduction to the discipline of Biomedical Informatics from the perspective of research in the field. } \\
\text { Lectures cover application domains and themes, active research topics, and evaluation methodologies }\end{array}$ \\
\hline $\begin{array}{l}\text { Clinical Information } \\
\text { Systems and Databases }\end{array}$ & $\begin{array}{l}\text { This course is taught using a combination of didactic and extensive programming exercises. Students } \\
\text { receive an introduction to distributed systems, networking computing concepts, and clinical databases. } \\
\text { They discuss architectural considerations in the design of clinical information systems, and program } \\
\text { examples of all of the above components of architecture. The VUMC clinical database architecture is } \\
\text { used as a case study for this course. }\end{array}$ \\
\hline
\end{tabular}

\section{Core Competency Courses}

The core competency courses include courses to enhance students' knowledge about biomedical science, informatics methods, and research methods. The goal of this requirement is to provide students with knowledge that is essential to complete applied and theoretical research. Table 2 outlines the breadth of courses students may take in these core areas. Course requirements in each area may be waived according to student background. In general, students matriculate with experience in at least one of the core competency areas.

Table 2. Examples Core Competency Courses*

Biomedical Science

- Introduction to Disease Processes

- Biology

- Biochemistry

- Advanced genetics

- Ethics and Medicine
Informatics methods

- Advanced Biomedical Informatics Algorithms

- Advanced Biomedical Informatics

- Data Structures \& Algorithms

- Managing Information Technology Projects

- Database Management Systems

\section{Research methods}

- Biostatistics

- Clinical trials

- Intermediate Statistics for the Health Sciences

- Qualitative Research Methods

- Mathematical Statistics

*Selected examples from dozens of courses that potentially fulfill requirements in each core area. 


\section{Elective Courses}

In addition to completing applicable courses from the two areas above, students may enroll in elective courses to obtain in-depth knowledge in the student's research area or in any other area of interest. In general, electives may be chosen from the entire catalog of courses offered by Vanderbilt's Graduate School.

\section{Other Degree Requirements}

Trainees who pursue a $\mathrm{PhD}$ degree are required to complete a MS degree. The MS thesis requirement can be waived and replaced by additional didactic course work for trainees interested in certain specialization areas that may require trainees to acquire indepth knowledge. PhD students take additional course work and have to pass a written and oral exam to obtain $\mathrm{PhD}$ candidacy status.

All MS and PhD students submit a formal thesis proposal. Students usually develop this proposal after substantial preliminary work under the supervision of a mentor in the department or elsewhere in the University. Thesis projects typically focus on one of six main areas in which our faculty has expertise:

- Decision Support Systems and Medical Decision Sciences

- Evidence-Based Practice Informatics

- Health Policy Informatics, Management, and Administration

- Clinical Systems

- Bioinformatics for Molecular Medicine

- ClinicalBioinformatics

Students are allowed to complete research for their $\mathrm{MS}$ and $\mathrm{PhD}$ projects on different topics (e.g., a clinical informatics project for the MS and a bioinformatics project for the $\mathrm{PhD}$ ). Typically, MS projects are designed to be a component of a larger ongoing project within the department, with specific aims that are able to be fully realized by the student. In contrast, doctoral projects may or may not be components of larger efforts, and are designed to not only capitalize on the strengths of the student and the University, but also to provide a foundation on which a graduate can build a career in biomedical informatics. Student research topics may also focus on an area outside of the seven areas defined above, such as economical aspects related to informatics in health care.

The program heavily emphasizes the written and oral presentation and communication of research findings to the biomedical informatics community during the entire program of study.

\section{Combined degree programs}

MD-PhD Program: DBMI participates in Vanderbilt's MD-PhD combined degree program, the Medical Scientist Training Program (MSTP). The MSTP student typically take 7 to 8 years to complete requirements for both degrees (MD and PhD in Biomedical Informatics).

Clinical Scientist Program: Residents interested in biomedical informatics and an academic career can enter the Clinical Scientist Program in which they start their research fellowship in the third year of residency training. During the fellowship trainees may start working on an MS degree in biomedical informatics.

Combined MS/PhD programs: Combined programs can be arranged and enable trainees in highly interdisciplinary fields to obtain the biomedical informatics training needed for their $\mathrm{PhD}$ degree in a different department. Students in combined programs will also have an interdisciplinary committeeincludingmembers from involved departments. An MD/MS in Biomedical Informatics program has also been set up by the School of Medicine.

\section{Post-Doctoral Fellowship Training in Biomedical Informatics}

The goal of the non-degree, postdoctoral fellowship program is to allow trainees to perform in-depth studies in a specialized area and expand their previous skills. Trainees in the nondegree program gain practical and theoretical knowledge and skills in one or a few biomedical informatics areas. For students with previous background or skills in biomedical informatics or a related field (such as those with an undergraduate rigorous major in computer science coupled with completed MD and residency training), the DBMI Training Program allows an "applied research fellowship" that prepares the trainee for a career in biomedical informatics. The nondegree program trainee develops and completes a substantial research project (with the goal of publication rather than a degree), but only takes a limited number of graduate courses (one per semester at most) specifically in areas of identified weaknesses or of career interests. The set of skills acquired during the period of the nondegree program depend on the length of study and the student's declared area of the interest and include, but are not limited to: (1) Technical skills: project planning, project management, project documentation (technical and scientific documentation, e.g. technical reports), project evaluation, and technical presentations of projects; (2) Organizational and personal skills: team building, team management, Interpersonal skills, effective communication, and time management; and, (3) Academic/scientific skills: knowledge about professional and ethical conduct; scientific writing (may include grant writing); scientific planning and evaluation of projects; publication and presentation at scientific meetings; knowledge about biomedical informatics organizations and agencies; knowledge about funding agencies and 
opportunities in biomedical informatics; and In-depth knowledge about the literature and publications pertinent to the project.

\section{Chief Information Officer Track}

Through the leadership of Drs. William W. Stead and Nancy M. Lorenzi, DBMI faculty members who are acknowledged world-wide for their skillsinmanagement and organizational change, DBMI plans to develop by Fall 2004, as part of its degree program, a track geared specifically to train future informatics leaders of health care organizations. Individuals trained in the CIO track will be able to understand: (1) Complex Health Care Organizations Today: Analyze relationships between organization structure and performance, including clinical processes and outcomes; Understand alternative organizational strategies and their operational implications; Understand the complex nature of organizations and how decisions are made; (2) Information Systems and Informatics; (3) Health Care: Understand the coordination and control of clinical functions in health organizations; Understand how to develop and apply criteria for assessing organizational and clinical performance; (4) Leadership and Motivating Individuals: Understand leadership styles and develop a personal leadership perspective; Understand the changing role of the manager in health institutions; Understand decision making in health care organizations; Develop personal leadership skills for the success; and, (5) Change and Transformation Strategies: Develop and implement change strategies to transform organizations.

\section{Medical and Undergraduate Student Education}

Medical and undergraduate students with an interest in Biomedical Informatics have the opportunity to complete a three-month rotation that usually includes an applied research project. This has been an extremely popular summer elective for both Vanderbilt Medical Students and Meharry Medical and Dental students, as well as for undergraduate students from around the United States. In 2004, the Vanderbilt University School of Medicine will introduce an innovative immersion experience, called the Emphasis Course, into the first and second year medical student curriculum. The Emphasis Course is designed to provide incoming medical students with an invaluable opportunity to pursue their existing or unexplored intellectual passions in order to make Vanderbilt students among the best-educated and most well-rounded physiciansleaders and scholars in medicine. Students will spend roughly one day per week during the first two years of medical school pursuing a specific project within one of many areas of interest. These include:

clinical and biomedical informatics laboratory based biomedical research

- health services research and policy patient-oriented research

education

medical humanities

community outreach and health initiatives

- law

The Emphasis Course will begin with a lecture series during the first semester of the first year that will serve as an introduction to the main areas of interest. After the first semester, students spend the next three semesters, and possibly the summer after first year, exploring a chosen topic of interest. By giving students two years to investigate this area of interest, the intention is that the Emphasis program will achieve meaningful student-mentor relationships. Students will beexpected to develop a product, likely to be similar in scope to but less advanced than a Master's project, as a measure of completion of the program. The pro- gram provides an incentive to students to have a tangible accomplishment by the time of graduation from Vanderbilt. Early surveys of students have suggested that biomedical informatics will be the preferred area of Emphasis for $10 \%$ of the student body.

One of the more difficult challenges facing the field of biomedical informatics is how to increase the number of underrepresented minorities in the field. Issues such as healthcare disparities and cultural barriers to the adoption of technology are among many that are not well-studied by researchers in ourfield. VanderbiltUniversity Medical Center is fortunate to have a strong relationship with Meharry Medical College, whose medical and dental programs attract predominantly African American and other underrepresented minority students. Each year, DBMI faculty are invited to teach Meharry students about biomedical informatics. For the last two summers, this program has resulted in many Meharry students completing 3-month summerinternship rotations with DBMI. Vanderbilt and Meharry are extremely optimistic about this relationship. This year, DBMI accepted its first Meharry graduate as a post-doctoral fellow concentrating in bioinformatics.

\section{Conclusions}

Over a decade, the Biomedical Informatics Training Program at Vanderbilt has rapidly evolved from a small, primarily service-oriented entity to a key stakeholder within Vanderbilt's clinical and research enterprise. The leadership and faculty of Vanderbilt University Medical Center view Biomedical Informatics as a primary and essential contributor to its global vision of providing high quality of care for patients and enhancing its biomedical research capabilities. The DBMI faculty have expertise in a broad spectrum of areas. The DBMI faculty 
and their trainees fuel creative and important ideas for both basic and applied research. The Vanderbilt Informatics Center pursue an interdisciplinary approach that provides the basis to forge long-term collaborations and relationships among investigators from different disciplines. The program is based on a close integration among the educational model, the research, and the operational strategies and business of the institution. The unique strategic alignment and close interactions at Vanderbilt form the foundation for trainees to pursue significant and meaningful research opportunities and to be directly educated by national and international leaders in the field of biomedical informatics — ranging from the molecular level, to individual patient care, to entire populations. This framework allows trainees on different educational levels and with a variety of research interests to prepare for careers in academia or industry and experience the field of biomedical informatics from both basic and applied perspectives.

\section{Acknowledgments}

Vanderbilt's biomedical informatics training program is supported by a grant from the National Library of Medicine (Grant No. T15 LM07450-02)

\section{APPENDIX 1:}

Faculty of the Department of Biomedical Informatics include (September 2003):

\section{Chair}

Randolph A. Miller, M.D. - Professor and Chair of the Department of Biomedical Informatics and Professor of Medicine; Associate Director of Informatics Center

\section{Vice-Chair}

Kevin B. Johnson, M.D., M.S. Associate Professor and Vice Chair of Biomedical Informatics, Associate

Professor of Pediatrics

\section{Professors}

Nunzia B. Giuse, M.D., MLS Associate Professor of Biomedical Informatics; Director of the Annette and Irwin Eskind Biomedical Library

Nancy M. Lorenzi, MLS, Ph.D. Professor of Biomedical Informatics; Assistant Vice Chancellor for Health Affairs

Judy Ozbolt, Ph.D., R.N. - Independence Foundation Professor of Nursing and Professor of Biomedical Informatics

William W. Stead, M.D. - Professor of Medicine, Professor of Biomedical Informatics, Director of the Informatics Center, Associate Vice Chancellor for Health Affairs, Assistant to the Chancellor for Informatics and Chief Information Architect for the University

\section{Associate Professors}

Steven H. Brown, M.D., M.S. Associate Professor of Biomedical Informatics; Director, Compensation and Pension, Examination Project, Veterans Health Affairs/Veterans Benefits Affairs; Dept. of Veterans Affairs

Dario Giuse, Dr. Ing. - Associate Professor of Biomedical Informatics and Computer Science; Associate Director of Informatics Center

Edward K. Shultz, M.D., M.S. Associate Professor of Biomedical Informatics; Director of Informatics Technology Integration, Informatics Center

\section{Assistant Professors}

Constantin F. Aliferis, M.D., Ph.D. Assistant Professor of Biomedical Informatics; Director of Discovery Systems Laboratory

Dominik Aronsky, M.D., Ph.D. Assistant Professor of Biomedical
Informatics and Emergency Medicine; DBMI Director of Training Programs

Erik Boczko, Ph.D., Ph.D. - Assistant Professor of Biomedical Informatics

Mary E. Edgerton, M.D., Ph.D. Assistant Professor of Pathology and Biomedical Informatics

Shawn Levy, Ph.D. - Assistant Professor Molecular Physiology \& Biophysics and Biomedical Informatics; Director of the Vanderbilt Microarray Shared Resource

Anderson Spickard, III, M.D., M.S. Assistant Professor of Medicine and Biomedical Informatics

Ioannis Tsamardinos, Ph.D. - Assistant Professor of Biomedical Informatics

Russ Waitman, Ph.D. - Assistant Professor of Biomedical Informatics

\section{Instructors}

Fern FitzHenry, Ph.D., R.N. Instructor, Biomedical Informatics

William Gregg, M.D., M.S.-Instructor of Biomedical Informatics

S. Trent Rosenbloom, M.D., M.P.H. -Instructor of Biomedical Informatics and Clinical Nursing

\section{References}

For a current list of references, please refer to the department's web site: www.mc.vanderbilt.edu/dbmi

Address of corresponding author: Dominik Aronsky, MD, PhD

Dir. of Graduate Studies

Dept. of Biomedical Informatics

Eskind Biomedical Library

2209 Garland Ave

Nashville, TN 37232-8340, USA

Tel.: $\quad+16159361068$

Fax: $\quad+16159361427$

E-mail: dominik.aronsky@vanderbilt.edu 